Meta

Journal des traducteurs

Translators' Journal

\title{
L'enseignement de la traduction juridique : une formation technique et universitaire
}

\section{Michel Sparer}

Volume 33, numéro 2, juin 1988

L'enseignement de la traduction au Canada - Teaching Translation in Canada

URI : https://id.erudit.org/iderudit/004205ar

DOI : https://doi.org/10.7202/004205ar

Aller au sommaire du numéro

\section{Éditeur(s)}

Les Presses de l'Université de Montréal

\section{ISSN}

0026-0452 (imprimé)

1492-1421 (numérique)

Découvrir la revue

Citer cet article

Sparer, M. (1988). L'enseignement de la traduction juridique : une formation technique et universitaire. Meta, 33(2), 319-328.

https://doi.org/10.7202/004205ar d'utilisation que vous pouvez consulter en ligne. 


\section{L'ENSEIGNEMENT DE LA TRADUCTION JURIDIQUE : UNE FORMATION TECHNIQUE ET UNIVERSITAIRE}

MiChel SPARER

Québec, Québec

Comment peut-on enseigner la traduction juridique?

Situons pour commencer certaines réalités ainsi que les limites de notre propos.

- On peut traduire des textes juridiques.

- Il n'est pas absolument indispensable d'être juriste pour faire de la traduction juridique.

- Il est préférable de l'être.

On peut enseigner les techniques de cette profession : je le fais depuis 12 ans.

Ces quelques paragraphes traiteront de l'enseignement de la traduction juridique sous l'éclairage particulier de la traduction des textes de lois et de règlements. Plutôt que d'examiner par le menu un plan de cours ou un syllabus, commençons par nous intéresser à certains grands axes de ce que devrait être un cours de traduction juridique. Nous pourrons ensuite, dans l'ordre des moyens, tenter de qualifier ou au moins de mettre en rang de priorité les divers éléments de cet enseignement.

Sans aller dans le détail, citons par ordre décroissant d'importance cinq priorités dans les sujets susceptibles d'intéresser la formation des étudiants en tenant pour acquis qu'ils ont déjà, à ce stade, reçu une formation en traduction générale.

- Description élémentaire du domaine juridique

- Présentation du système juridique

- Étude des règles de rédaction et d'interprétation

- Acquisition du savoir-faire technique proprement dit

- Détermination théorique et expérimentale de la marge de manœuvre du traducteur juridique

Tels seront les principaux points abordés dans le présent article qui se terminera par un schéma décrivant les principales phases de notre cours, les diverses formules pédagogiques qui leur correspondent ainsi que le temps minimum qu'on devrait consacrer à chacune d'elles.

\section{LA DESCRIPTION ÉLÉMENTAIRE DU DOMAINE JURIDIQUE}

Il est capital avant toutes choses de faire en sorte que l'étudiante et l'étudiant puissent comprendre ce qu'est le droit. On ne peut leur lancer le défi de voyager dans ce domaine sans leur avoir propoé un portrait d'ensemble du domaine juridique. Livré(e) à lui ou à elle-même, le traducteur et la traductrice finiraient bien par découvrir que le droit est un domaine composite. Pourtant, nous sommes convaincus qu'il faut les précéder dans ce cheminement et leur présenter le droit en premier lieu pour ce qu'il est : un ensemble complexe où on retrouve des grands domaines : droit privé, droit public, ainsi que des spécialités de plus en plus nombreuses, tel le droit administratif, le droit fiscal, le 
droit de l'espace, le droit linguistique, le droit de la guerre, etc., la liste ne cesse de s'allonger. Il est relativement aisé d'illustrer à l'aide d'exemples le type de règles qu'on retrouve dans chacune de ces spécialités, ainsi que la mission sociale à laquelle ces règles répondent.

\section{PRÉSENTATION DU SYSTÈME JURIDIQUE}

La description de type élémentaire n'est pas suffisante. Elle ne sert qu'à situer les masses en présence et à indiquer à quel domaine de l'activité sociale, politique ou économique s'applique le droit. Reste la seconde étape, la plus importante, celle qui consiste à présenter la structure et la dynamique du droit et de ses éléments. Or, nous touchons là à un point essentiel : le droit constitue un système doté d'une dynamique. Ignorer la structure et la dynamique de ce système priverait les futurs traducteurs ou traductrices d'une perspective importante. Cela reviendrait à leur confier mille morceaux d'un cassetête sans leur montrer la figure à reproduire. Nous en ferions des ouvriers et ouvrières à qui on fait assembler les pièces d'un appareil dont jamais ils ou elles ne sauront à quoi il sert ni comment il fonctionne. Comment pourront-ils dans ces conditions connaître la conséquence d'une variation ou d'un oubli dans la séquence de fabrication qui leur est impartie ? Des juristes eux-mêmes se plaignent de ce que le droit ne leur soit pas présenté de façon claire et systématique au tout début de leurs études 1 .

Le droit est un système sous la forme d'un ensemble organisé. La compréhension de cette structure de système est à notre avis indispensable à celui ou celle qui, abordant cette spécialité, aura le souci de comprendre ce qu'il ou elle fait, et par là même de connaître les conséquences de ses actes professionnels. Disons le tout net, il est dérisoire de s'aventurer dans le domaine juridique avec un espoir d'y être efficace et fiable, si on n'en connaît pas les lois de composition interne, pour emprunter au vocabulaire des mathématiques modernes. Voilà pourquoi, il convient d'indiquer en priorité aux étudiantes et étudiants que le droit répond au moins à six caractéristiques.

1. Le droit est un constat formel de choix sociaux. Les textes et règles doivent être compris en fonction du but poursuivi par le législateur.

2. Le droit est un ensemble d'éléments interactifs : la loi suscite de la jurisprudence et des débats de doctrine qui, à leur tour, inspirent le législateur.

3. Le droit connaît diverses sources : la loi, la coutume, la jurisprudence, la doctrine, etc.

4. Le droit est une discipline qui comporte des contraintes méthodologiques spécifiques.

5. Les règles de droit sont hiérarchisées, c'est-à-dire que de la constitution au décret, elles doivent être compatibles avec la règle de droit qui leur est supérieure dans cette hiérarchie.

6. Le droit écrit recourt à des règles de rédaction et d'interprétation qui forment, notamment pour le traducteur, un code linguistique incontournable.

Ayant affaire à ces traducteurs au Québec par exemple, il convient également de les avertir du caractère hybride du système juridique puisqu'il puise ses sources, mais aussi ses méthodes, dans deux grands systèmes occidentaux : le droit romanogermanique et le common law. Cet aspect qui, d'ailleurs, n'a pas encore livré tous ses mystères aux juristes eux-mêmes, est particulièrement délicat car il expose l'étudiant à se représenter de façon erronée certaines démarches. Exemple : traduisant de l'anglais au français, passe-t-on du common law au droit civil ? Certes non. Et pourtant, même s'il existe un vocabulaire anglais du droit civil et un vocabulaire français du common law, la façon qu'ont les francophones et les anglophones d'envisager l'organisation des rapports sociaux n'est pas la même. De plus, leur façon respective de formuler les règles de droit variera considérablement. Qu'on songe, par exemple, à la frontière entre le dit 
et le non-dit en nous rappelant que les règles de droit ne sont pas toutes exprimées et que le non-dit n'est pas forcément du non-droit. Exemple : lorsqu'une disposition indique qu'une personne a droit au remboursement d'une somme par une caisse de retraite, le juriste de droit romano-germanique déduira que la caisse doit la lui rembourser. Le juriste de common law, lui, jugera utile de le mentionner expressément. Ainsi, cette obligation corollaire existera sans être exprimée pour les uns, alors que pour les autres, elle ne saurait exister sans être exprimée. Ceci nous amène à des distinctions quant aux réflexes culturels, sujet que nous avons déjà abordé à maintes reprises ${ }^{2}$.

Il faut un certain temps pour expliquer à nos étudiants que les fonctions et méthodes du droit législatif par exemple sont différentes selon qu'on se situe dans un système de common law ou dans un système de droit romano-germanique. Pour un juriste de common law, le droit est en quelque sorte immanent : les tribunaux nous le révèlent et la législation permet d'y déroger. Pour un juriste de droit français, par contre, le droit est de préférence écrit. Il peut précéder la réalité : la loi, source principale, peut alors tracer les grandes lignes de l'organisation des rapports sociaux en dehors de toute orientation déterminée au préalable par les tribunaux.

Sans une présentation sérieuse de ces aspects, comment un traducteur ou une traductrice francophone, non juriste de surcroît, pourront-ils comprendre la portée et même l'objet des lois rédigées en anglais et dont des passages entiers sont consacrés à égrener des dérogations au common law ? Aussi, est-il capital de décrire à l'étudiant et à l'étudiante les sources du droit dans l'un et l'autre système, d'en comparer la hiérarchie et de leur montrer à quelles différences les disparités des deux systèmes obligent.

Le traducteur qui aborde un texte sans savoir le situer dans ce système risque de commettre des erreurs et de s'attirer de son client ou de son réviseur des commentaires qu'il ne comprendrait sans doute pas.

\section{L'INTERPRÉTATION}

Ayant présenté le droit en tant que système, il est important d'indiquer aux étudiantes et aux étudiants que le droit écrit fait l'objet de règles d'interprétation. Ces règles, développées de manière formelle, surtout dans les pays de common law, visent à orienter le juge dans sa compréhension du droit qu'il doit appliquer et à lui permettre de découvrir l'intention du législateur ${ }^{3}$. Pour beaucoup, ces règles recourent à une logique que ne renieraient point nombre de lecteurs, quelle que soit leur région. Ainsi, la règle indiquant que dans une énumération ce qui est exprimé exclut ce qui ne l'est pas, formalise un réflexe logique auquel nombre de citoyens adhéreraient sans peine. Par contre, la règle très utile voulant que deux termes différents font référence à deux concepts différents, principe qui bannit la synonymie, n'est pas un réflexe aussi répandu. N'y a-t-il pas d'ailleurs en littérature un usage qui consiste à recourir à des synonymes pour éviter les répétitions?

À côté des règles d'interprétation auxquelles d'ailleurs on ne recourt qu'en cas d'obscurité ou de contestation du sens d'une disposition, on trouve des dispositions d'interprétation dont les pays de common law font grand usage : les définitions. La législation de facture anglaise contient en effet un nombre important de définitions, situées en majeure partie en tête des lois. Ces définitions constituent à elles seules un système d'interprétation, puisqu'elles assignent un sens fixe et inhabituel à des termes fréquemment employés dans le texte de lois. Nous avons déjà à maintes reprises soulevé le caractère contestable et dangereux de ce procédé qui n'est la plupart du temps qu'une solution boiteuse apportant autant d'inconvénients que le problème qu'elle est censée résoudre. Par ces définitions en effet, le légiste de common law tente, la plupart du temps, de décrire le champ d'application du texte qu'il rédige ou encore d'indiquer des variations du régime juridique s'appliquant à telle ou telle réalité. Ces définitions parfois incohérentes 
ont pour effet de fausser le lexique en donnant à des mots des sens artificiels de nature à insécuriser le lecteur, qu'il soit avocat, juge ou traducteur, puisqu'ils ne peuvent plus compter sur leur compréhension de la langue courante pour lire les lois et règlements ${ }^{4}$.

Le nombre important des règles d'interprétation ainsi que leur complexité nous conduisent à penser qu'il est important sinon indispensable que les traducteurs qui seront amenés à produire des textes juridiques, soient mis en contact avec la théorie et l'expérimentation du système d'interprétation. Car enfin, c'est à l'aulne de ces règles que se pèsera le sens de ce qu'ils écrivent.

Avant d'amener les étudiantes et étudiants à acquérir un savoir-faire proprement dit, il est utile de synthétiser le tour d'horizon théorique et descriptif par une présentation du système en marche. À cet effet, depuis plusieurs années nous présentons aux étudiantes et étudiants le cheminement typique d'une règle de droit en prenant un exemple réel et si possible d'actualité.

Parlant de l'évaluation d'une situation appelant une intervention législative, nous expliquons à l'aide de textes les diverses phases qui se présenteront : décision de réforme, choix des modalités de réforme, rédaction d'un avant-projet de loi, adoption de l'avant-projet par le gouvernement, rédaction du projet de loi, consultations, débats, cheminement parlementaire, mise en application de la loi, phases administrative et judiciaire du contentieux d'application de la loi. On indiquera pour chaque phase les changements de nature et d'effet du texte, ainsi que les changements quant aux contraintes juridiques et linguistiques (rédaction, interprétation) dont se charge le texte en acquérant force de loi ou en étant interprété ou invalidé par les tribunaux.

\section{ACQUISITION DU SAVOIR-FAIRE PROPREMENT DIT}

Après avoir présenté le système juridique et expliqué le volet proprement linguistique de ce système que constituent les règles d'interprétation, le temps est venu pour les étudiants et les étudiantes d'acquérir un savoir-faire au plan traductionnel. Mais au risque de surprendre, nous pensons qu'à ce stade du cours il est prioritaire de mettre l'accent, encore une fois, sur la rédaction et ses techniques. Dans la séquence des acquisitions en matière de savoir-faire rédactionnel, mentionnons, sans les citer tous, quelques exercices particulièrement utiles.

En premier lieu, il s'agit de la simplification de texte du français au français.

Un félidé digitigrade préalablement victime d'une aspersion d'eau portée à un haut degré de température ne cessera d'éprouver la plus vive phobie rémanente à l'égard d'un liquide de même nature ramené à un niveau thermique inférieur ${ }^{5}$.

Il n'est pas nécessaire d'aller aussi loin dans le pastiche. Nous avons tous déjà rencontré en français des textes ampoulés ou insuffisamment rédigés ou encore des textes dont le style abusif donne de l'auteur une image qu'il n'a sans doute pas souhaitée et qui détourne très efficacement le lecteur de la simple compréhension du message.

La tension superfonctionnelle dans un régime de type fédéral ne s'analyse donc pas comme la conséquence du seul malfonctionnement conjoncturel du système ; elle lui est en quelque sorte inhérente. Le point par-dessus tout capital à rappeler consiste à n'y pas voir l'effet du choc, toujours virtuel, de deux principes de légitimité s'affrontant à la verticale. Avant d'en arriver là, il y a eu d'abord perception, exacte ou non, dans une politique fédérée que sa sécurité spécifique est insuffisamment assurée par son intégration à la politique fédérale. Aussi, l'État fédéré peut-il prétendre assumer "sa " part de la sécurisation externe, qui est normalement du ressort principal de l'État central. Dans l'État fédéral, le processus de sécurisation comme celui de la légitimation, est donc susceptible de se dérouler aux deux degrés des États fédérés et de l'État central - tout superfonctionnels, c'est-à-dire unificateurs, que soient ses vastes processus. 
Davantage il conviendrait peut-être de dire en forme synthétique que la tension superfonctionnelle propre à la structure même de l'État fédéral en totalité est toujours produite par les ressorts interagissants de la légitimation et de la sécurisation dans cet ensemble complexe à deux degrés.

L'histoire récente du Québec présente deux cas illustrant dans le concret ces propositions d'élaboration théorique abstraite sur les processus superfonctionnels de légitimation et de sécurisation : les relations extérieures au Québec depuis 1961 pour cette dernière superfonction et la crise d'octobre 1970 pour celle de la légitimation ${ }^{6}$.

Il est certain que nous ne proposons pas ces deux textes à nos étudiants et étudiantes. Nous leur soumettons plutôt dans un premier temps, des passages de textes législatifs dont la seule difficulté réside dans une rédaction trop complexe. Plus précisément, on commence par faire des exercices de simplification de l'expression par la transformation d'articles, de paragraphes ou de phrases en langue française. L'exercice, on l'aura deviné, vise à donner des réffexes rédactionnels à l'étudiant et à l'étudiante. Le but est de leur donner ainsi des habitudes et aptitudes qui influenceront jusqu'à leur manière de lire et de comprendre les textes. Il est en effet possible par ces exercices d'amener l'étudiant et l'étudiante à percevoir dès la lecture les sources et causes de l'obscurité ainsi que les chemins de la clarification.

En second lieu, une fois ces mécanismes acquis, il est possible de procéder à un autre type d'exercice, c'est-à-dire la simplification directe, de l'anglais au français. Le traducteur part donc d'un texte de départ obscur pour aboutir grâce à ces nouveaux réflexes, à un texte d'arrivée plus clair.

En troisième lieu, nous proposons à l'étudiant et à l'étudiante, un exercice qui consiste à leur demander de réviser la version française de textes bilingues. Il est bien sûr trop tôt à ce stade de la formation pour transformer notre élève en réviseur ou réviseure de niveau professionnel. L'exercice est pourtant très efficace si nous en jugeons par les résultats obtenus depuis dix ans. L'étudiant et l'étudiante sont amenés à examiner un texte de manière critique. Ils s'attachent alors à ce qui est le plus apparent, c'est-à-dire le style, la grammaire, l'orthographe, la ponctuation, le vocabulaire puis, en général, à " la fluidité " du texte, à sa lisibilité. À l'aide des indications données par le professeur quant à la structure et à la longueur des phrases, à l'agencement des idées, aux connotations ainsi qu'aux variations d'éclairage, le réviseur en herbe peut alors par cet exercice, isoler d'une part, les effets de méthodes inadéquates et d'autre part, les méthodes inadéquates en question. Par ailleurs, cet exercice de révision et éventuellement de réécriture a un effet latéral non négligeable. En effet, l'étudiant et l'étudiante sont habituellement des observateurs sévères. Ayant fait preuve de sévérité dans leurs révisions, ils se feront un point d'honneur à ne pas encourir eux-mêmes les reproches dont ils ont accablé le traducteur du texte qu'ils révisent. Enfin, au-delà de la critique ponctuelle, cette révision les amène à s'interroger sur les contraintes qui ont pu s'exercer sur le traducteur du texte. Une étape importante de cet exercice est celle où l'étudiant et l'étudiante examinent le texte de départ pour faire le bilan des contraintes qui peuvent être contournées et de celles qui ne peuvent l'être.

Onze années d'enseignement de cette matière nous permettent de croire que tels sont les préalables qui donnent à l'étudiant la capacité d'aborder en connaissance de cause les textes juridiques et plus particulièrement, les textes de lois et de règlements. Il n'est pas question de négliger ou de minorer l'importance des autres sujets qui constituent la suite du programme que nous avons bâti. Toutefois, il s'agit là d'acquisition de techniques qui intéressent moins l'essentiel des textes qui leur sont soumis. Il s'agit, sans 
citer tous ces sujets, de la sculpture des phrases et des articles, du traitement des phrases longues, de la dimension du contenu et de la structure de l'article, de l'articulation selon l'éclairage (par exemple, la présentation d'une règle sous la forme d'une obligation, d'une condition ou d'une interdiction, l'éclairage différant selon qu'on aborde la règle en allant du général au particulier ou à l'inverse).

\section{LA MARGE DE MANOEUVRE DU TRADUCTEUR}

En laissant de côté la description chronologique du cours, il convient d'aborder une démarche importante pour la formation de l'étudiant et de l'étudiante, mais aussi pour sa pratique professionnelle : il s'agit de l'exploration exhaustive de la marge de mancuvre du traducteur en matière de traduction et plus précisément en matière de choix rédactionnel. Car en fin de compte, c'est bien de cela qu'il s'agit : que peut se permettre le traducteur lorsqu'en s'écartant des structures du texte de départ pour produire un texte d'arrivée plus simple et plus clair, il rompt le parallélisme des formes qui a si longtemps fait et fait encore ça et là le critère immédiat de vérification d'une traduction juridique exacte?

La réflexion universitaire et la pratique nous enseignent qu'il existe des bornes à ne pas dépasser dans la liberté qu'on prend avec les formes, l'éclairage et bien sûr, avec le sens du texte. Toutefois, on peut affirmer que ces bornes peuvent être repoussées plus loin qu'on ne le pense, pourvu qu'on s'applique à considérer avant tout le but poursuivi par le législateur. Le but que poursuit le législateur bilingue n'est pas de reproduire d'une langue à l'autre une morphosyntaxe ou une sémantique figées, mais est avant tout de reproduire les effets de droit, c'est-à-dire les conséquences juridiques du texte de départ. Cela constitue la mission première et essentielle du traducteur. Aucun compromis, aucune modulation n'est possible quant à la transmission des effets de droit. Ce principe doit être présent à l'esprit de l'étudiante et de l'étudiant puis à celui de quiconque concourt à la réalisation de lois ou de règlements bilingues ou multilingues.

Pourtant, la manière différente dont les francophones expriment leurs droits ne peut et ne doit être oubliée ni remise totalement en cause dans ce processus, même s'il s'agit de traduire de l'anglais au français un texte de common law ou du français à l'anglais un texte de droit civil. S'il est indispensable de retrouver à gauche et à droite de la page des règles portant les mêmes conséquences juridiques, on ne doit pas pour autant y sacrifier ce qui fait la spécificité de la langue et de la culture de ceux à qui la traduction est destinée. Ainsi, la morphosyntaxe, le découpage des concepts, la frontière usuelle entre le dit et le non-dit, l'agencement des idées sont entre autres, des aspects du texte qui sont susceptibles de connaître des transformations au cours du processus traductionnel. Les destinataires du texte d'arrivée n'auront pas forcément les mêmes usages que ceux du texte de départ à cet égard. En 1986, on ne peut concevoir que le destinataire du texte d'arrivée doive pour comprendre ce texte, adhérer aux choix linguistiques et culturels qui marquent la forme du texte de départ. Chaque langue et chaque culture a sa manière d"exprimer les concepts, d'articuler les idées, d'exprimer ou de sous-entendre telle ou telle partie de la séquence logique.

Bien sûr, la détermination de la marge de manœuvre du traducteur est une démarche complexe qui ne peut être entièrement faite lors des études universitaires. Elle se poursuit et prend son sens lors de l'expérimentation pratique en circonstances professionnelles réelles. Toutefois, la démarche doit avoir été entreprise et bien éclairée dès le cours universitaire. Explorer la marge de manœuvre du traducteur vise à trouver les moyens d'éliminer les contraintes inutiles et nuisibles à l'intégrité linguistique, voire culturelle, du texte d'arrivée. À cet égard, nous ne pensons pas qu'à lui seul le contact des traducteurs avec les réviseurs - formés autrement, ailleurs ou à une autre époque -- favorisera l'élimination de ces contraintes. L'objectif de sécurité juridique donne en- 
core lieu à trop de malentendus chez certains. Il paralyse la réflexion et provoque un phénomène de mise en retrait des compétences linguistiques du traducteur et de son réviseur. On se méfie de tout écart quant au parallélisme formel. Pour des raisons bien compréhensibles, les choix structurels, stylistiques, terminologiques, voire grammaticaux se font encore par rapport au texte anglais, c'est-à-dire qu'ils sont marqués par la manière dont le légiste anglophone a conçu et articulé des règles qu'il a jugé bon de rendre expresses.

Compte tenu des impératifs de la traduction juridique, il est important de porter à la connaissance de ceux qui vont traduire vers le français ou vers l'anglais les travaux que les auteurs anglophones ont consacrés à la remise en cause de leur propre pratique rédactionnelle. Cette remise en cause est vigoureuse et inventive ${ }^{7}$. De plus, elle a pour objectif de rendre les textes plus proches de la capacité de compréhension du justiciable. Pouvons-nous ajouter que cet objectif est voisin du nôtre qui est de faire en sorte que le texte d'arrivée soit, selon le cas, en bon français ou en bon anglais. Les deux langues officielles du Canada ne sont pas l'anglais et le bilinguisme. Ce ne sont pas non plus l'anglais et une langue de synthèse qui serait à la remorque des contraintes de la langue de départ. Au contact des auteurs anglais, canadiens anglais et américains, les futurs traducteurs apprendront plusieurs choses intéressantes.

- Les juristes anglophones ne sont pas tous satisfaits de la rédaction ordinaire des textes relevant du common law.

- Ils envisagent - et donc considèrent possibles — des réformes d'envergure de leur propre pratique rédactionnelle anglaise ${ }^{8}$.

Nos futurs traducteurs seront amenés à s'interroger sur le fait que trop souvent encore, nombre de clients, de réviseurs ou de professeurs réclament qu'on maintienne la version française le plus près possible d'un texte anglais dont les procédés de rédaction sont critiqués et bientôt rejetés par les juristes anglophones eux-mêmes. La doctrine américaine ou canadienne anglaise aura tôt fait de fossiliser les querelles qu'à cet égard se livrent auteurs, traducteurs et réviseurs au Canada. Ces polémiques masquent des réalités premières comme celle qui veut qu'un texte bilingue soit écrit - encore une fois - en bon anglais et en bon français et qu'on ne sacrifie pas le français sur l'autel du parallélisme formel.

Bref, il convient de donner à nos élèves les connaissances et les méthodes qui leur permettront de mesurer de façon utile la liberté qu'ils peuvent exercer au profit de la qualité de leur texte d'arrivée. Wallace Schwab et moi-même tentons depuis longtemps d'élargir la marge de manœuvre des traducteurs en leur proposant des paramètres de rédaction faisant appel à leur intelligence et à leur sensibilité culturelle.

\section{CONCLUSION}

Telles sont les grandes lignes de ce qu'il convient à notre humble avis de proposer à nos étudiantes et à nos étudiants en traduction juridique. On les trouvera schématisées en annexe du présent texte. Peut-on imaginer faire tout ce chemin en 45 heures avec des étudiants qui, au début de la session, ignorent pour la plupart, ce qu'est une loi, une constitution, une convention et sont incapables de dire si l'État canadien est de forme républicaine, monarchique, dictatoriale ou autre? Allons-nous dire, par ailleurs, qu'il faut ajouter 700 heures et 40 crédits au diplôme de traduction ou en droit pour aboutir à une formation adéquate des professionnels de la traduction juridique? De notre point de vue, un professionnel n'est jamais trop bien formé. Les employeurs sont d'ailleurs plus exigeants depuis quelque temps. Ils ont remarqué par exemple qu'un nombre croissant de juristes se portent candidats à des postes de traducteurs. Certains en sont même venus à penser qu'on pourrait désormais exiger un diplôme en droit comme complément à la formation des traducteurs se destinant à la traduction juridique. D'où la ten- 
dance à imaginer des programmes, qui malgré toutes leurs qualités, relèvent de cette propension à tenir pour acquis la disponibilité des juristes pour la traduction. Or, cette disponibilité pourrait bien être éphémère. En effet, elle s'explique par la difficulté momentanée de trouver à s'employer dans les professions juridiques traditionnelles. Cette situation, faut-il le préciser, est purement conjoncturelle. $\grave{A}$ cet égard, on peut envisager deux hypothèses.

- D'une part, l'activité économique reprend durablement et l'essor retrouvé du secteur des services permettra aux juristes actuellement en disponiblité d'exercer leurs talents comme notaires ou comme avocats.

- La conjoncture économique continue d'être mauvaise et les étudiants entrant à l'université seront avertis de la faiblesse des débouchés en droit et choisiront d'autres branches.

Dans l'une comme dans l'autre hypothèse, il faut s'attendre à voir diminuer le nombre de juristes qui sont disponibles pour la traduction à titre d'activité principale. Faut-il fonder les conditions d'accès aux postes de traduction juridique sur le fait qu'il existe et existera toujours des personnes qui, déjà diplômées en droit, sont prêtes à acquérir une qualification supplémentaire pour accéder à la profession de traducteur ? L'avocat ou le notaire seront-ils toujours disposés à consacrer deux ou trois sessions supplémentaires pour devenir traducteurs juridiques, c'est-à-dire pour gagner - soyons concrets - $10000 \$$ de moins que s'ils étaient embauchés en tant que conseillers juridiques de l'État sans faire l'effort de ces trois sessions supplémentaires?

Pour rester dans des limites utiles et en attendant que les débouchés en traduction juridique attirent les juristes au point qu'ils feront les frais de deux ou trois sessions supplémentaires, on peut considérer comme possible - et non idéal - de donner en un bloc de 90 heures de programme intégré, les bases et le minimum d'expérience pratique qui permettent à un étudiant studieux et relativement doué de se présenter décemment devant un texte en circonstances professionnelles réelles et ce, dans une spécialité donnée de la traduction juridique.

Nous ne prétendons pas qu'en ces 90 heures, on peut rendre le traducteur polyvalent au point de lui ouvrir tout à la fois les portes du domaine législatif, du domaine judiciaire, les contrats ou encore les différentes branches du droit public moderne. Nous sortirons au moins du cadre trop restreint que nous offrent les 45 heures qu'on consacre d'ordinaire à la version juridique. Le programme proposé vise à permettre à ceux qui vont aborder de manière professionnelle le domaine juridique de le faire avec assurance ou, tout du moins, avec une pleine conscience des contraintes qu'impose le système. Ainsi, cette stratégie d'enseignement permet-elle d'éviter au futur traducteur les excès que constituent d'une part, une trop grande timidité quant aux textes et d'autre part, les imprudences, excès tous deux imputables à la méconnaissance des structures et contraintes du système. Si nous devions résumer en une formule le résultat attendu du programme proposé, nous pourrions dire qu'il a pour ambition de faire de nos étudiants et de nos étudiantes, de véritables professionnels, c'est-à-dire des intervenants qui comprennent ce qu'ils font et accèdent ainsi à l'autonomie, caractéristique principale du travail professionnel.

Notes

1. Lisette Savard (1984) : "L'introduction au droit : des étudiants insatisfaits ", Journal le Barreau 84, octobre. Extraits d'une étude intitulée l'Introduction au droit, Québec, 1982, 67 p. Mémoire présenté à la faculté de droit de l'Université Laval dans le programme de licence.

2. "Loi et héritage culturel ", M. Sparer et W. Schwab (1979) : les Cahiers de droit, Presses de l'Université Laval, Québec, mars ; "Pour une dimension culturelle de la traduction juridique ", M. Sparer (1979) : META, 24:1, les Presses de l'Université de Montréal, pp. 68-95; "La stérćophonie législative, facteur de haute infidélité ", M. Sparer (1981) : les Cahiers de droit, mars, Québec, Presses de l'Université Laval; 
Rédaction des lois, rendez-vous du droit et de la culture, M. Sparer et W. Schwab (1980) : Québec, Conseil de la langue française, $349 \mathrm{p}$.

3. P.A. Côté (1982) : Interprétation des lois, Yvon Blais, p. 3.

4. Rédaction des lois, rendez-vous du droit et de la culture, op. cit., $\mathrm{n}^{\circ} 2$.

5. Sven Sainderichin (1976) : Écrire pour être lu, Paris, Entreprise moderne d'édition, p. 34.

6. Gérard Bergeron (1984) : la Pratique de l'État au Québec, Québec Amérique, p. 217.

7. Cf. par exemple : Sir William Dale (1977) : Legislative Drafting : A New Approach, London, Butterworth R.C. Dick (1972) : Legal Drafting, Toronto, Carswell, 215 p., et bien d'autres encore.

8. R. Dick, op. cit., note 7 .

\section{ANNEXE}

\section{Synthèse d'une stratégie pédagogique pour le cours de traduction juridique} PHASE I

Présentation d'ensemble des réalités juridiques

\author{
Formule pédagogique \\ $24 \mathrm{~h}$ \\ Cours magistral \\ et lectures
}

Acquisition de techniques

\section{Formule pédagogique}

$50 \mathrm{~h}$

Cours magistral

Expérimentation et lectures

\section{Détermination de la} marge de manœuvre du traducteur

\section{Formule pédagogique}

Cet aspect est sous-jacent aux activités de la phase II mais devrait en plus faire l'objet de 15 heures de cours magistral, d'expérimentation et de lectures (doctrine et jurisprudence)
Description élémentaire du domaine juridique
Nature, structure et dynamique du système juridique
(Finalité, sources et méthodes du droit)
Éléments de logique juridique
Formalisme et interprétation

\section{PHASE II}

Simplification de textes (F-F, A-F)

Techniques de rédaction des lois

Application à la traduction et à la rédaction bilingue des techniques de rédaction et d'interprétation

\section{PHASE III}

Évaluation des virtualités et des conséquences des variations ou innovations rédactionnelles sur l'identité des effets de droit des textes bilingues 